\title{
PERIPHERAL NERVE BLOCK IN A PATIENT ON DUAL ANTI-PLATELET THERAPY
}

\author{
A. Tay ${ }^{1}$, B. Bellew ${ }^{1}$. \\ ${ }^{1}$ St Mary's Hospital- Imperial College Healthcare NHS Trust, Department of Anaesthesia, London, United Kingdom.
}

Important developments in the use of percutaneous coronary intervention $(\mathrm{PCl})$ in combination with coronary stent insertion have led to its widespread use. Dual antiplatelet therapy (DAPT) is commonly used to prevent stent thrombosis. Anaesthetists will increasingly encounter patients who have had recent $\mathrm{PCI}$ on DAPT presenting for non-cardiac surgery. Non-cardiac surgery within 4 weeks of $\mathrm{PCl}$, stenting and DAPT carry a high risk of major acute coronary events and haemorrhage ${ }^{1,2}$. 58 year-old male presented for excision of a large left forearm lesion less then 4 weeks post-PCl, with single bare metal stent and on DAPT. An exercise tolerance test demonstrated ST-depression at peak exercise. An ECHO showed good left ventricular function and no valvular disease. He had a previous lower limb deep vein thrombosis after previous knee surgery. The surgeon was keen to proceed with the surgery despite the risk of bleeding because of the risk of malignancy. The patient also wished to proceed with the surgery because it was causing him pain and psychological distress. Perioperative cardiac risks with GA were deemed to be higher risk compared to risks of bleeding associated with peripheral nerve blockade.

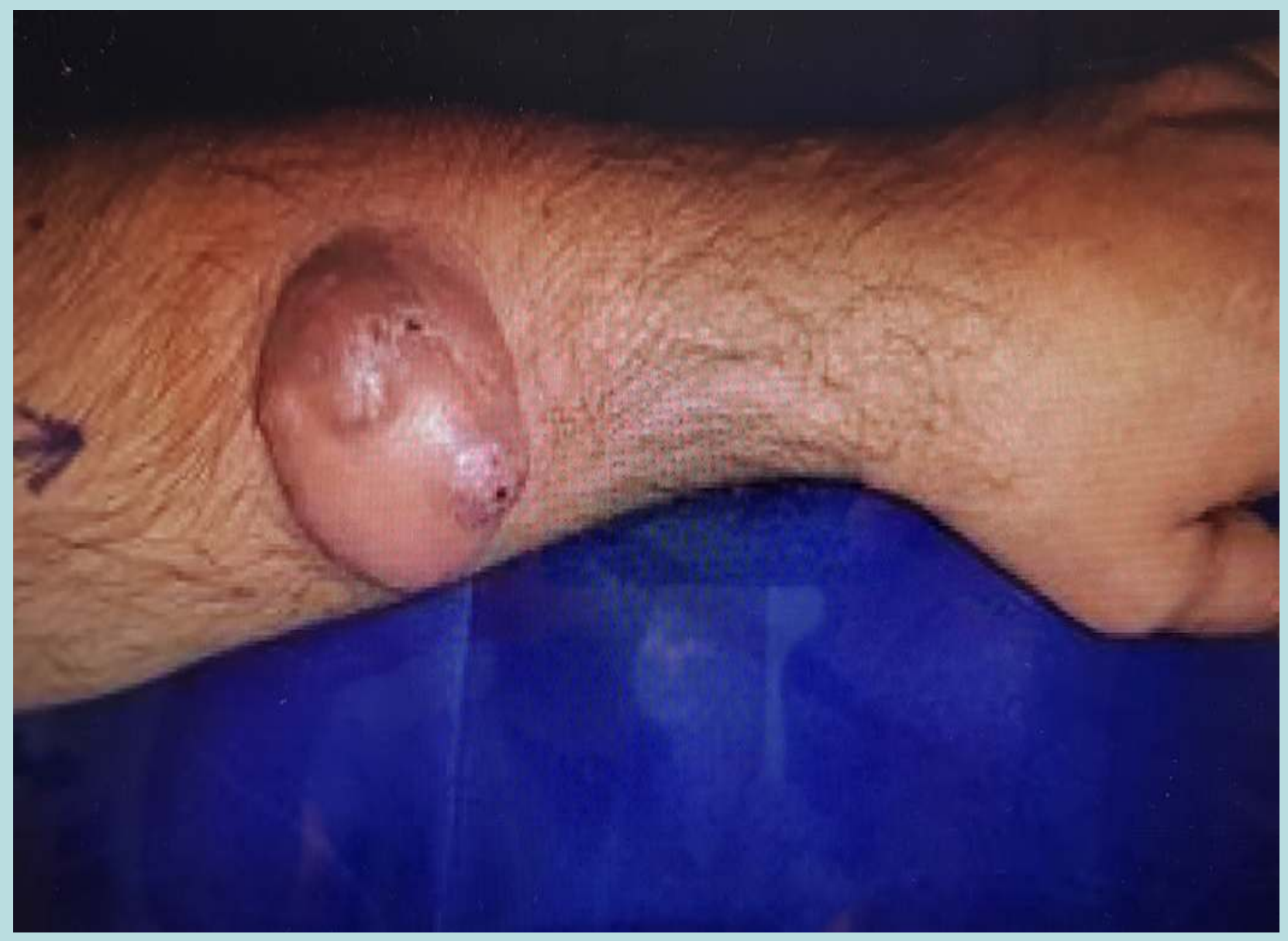

Ultrasound-guided supraclavicular brachial plexus nerve block was performed and using a propofol targetcontrolled infusion for sedation, good surgical conditions were obtained.

The management of patients with a recent coronary stent presenting for urgent non-cardiac surgery is an increasing clinical problem. Careful ultrasound-guided and Dopplerguided peripheral nerve blockade undertaken by an experienced operator may provide a useful alternative to a high risk $G A$.

References:

1. Barash P, Ahktar S. Coronary stents: factors contributing to perioperative major adverse cardiovascular events. $\mathrm{Br} \mathrm{J}$ Anaesth. 2010 Dec;105 Suppl 1:i3-15.

2. Newsome RT, Weller RS, Gerancher JC, et al. Coronary artery stents: II. Perioperative considerations and management. Anesth Analg. 2008 Aug;107(2):570-90. 